\title{
The deep Swire VLA field: faint radio populations
}

\author{
Veronica Strazzullo* ${ }^{\dagger}$ \\ National Radio Astronomy Observatory \\ E-mail: vstrazzu@nrao.edu \\ Maurilio Pannella \\ National Radio Astronomy Observatory \\ E-mail: mpannellenrao.edu
}

\section{Frazer N. Owen}

National Radio Astronomy Observatory

E-mail: fowen@nrao.edu

\begin{abstract}
We present a study of a $20 \mathrm{~cm}$ selected sample in the Deep SWIRE VLA Field, reaching a $5-\sigma$ limiting flux density at the image center of $S_{1.4 \mathrm{GHz}} \sim 13.5 \mu \mathrm{Jy}$. In a $0.6 \times 0.6$ square degrees field, we were able to assign a optical/IR counterpart to the vast majority (97\%) of the radio sources. The observed spectral energy distributions of these counterparts in up to 11 passbands from the NUV to $4.5 \mu \mathrm{m}$ were used to derive photometric redshifts and to constrain the stellar population properties of the sources. We focus on the radio sample in the redshift range $0.3<z<1.3$ where we estimate to be more than $90 \%$ complete in terms of counterparts and redshifts. The whole faint radio population in this redshift range was divided, based on the optical/NIR spectral energy distribution, in three classes of quiescent, intermediate, and starforming galaxies, in the expectation that quiescent galaxies have their radio flux dominated by AGN while the radio emission from starforming galaxies has a relevant contribution from star formation activity. We find that, as expected, the relative contributions of AGN and starforming galaxies to the $\mu \mathrm{Jy}$ population depend on the flux limit of the sample, however at all flux levels a significant population of intermediate, "green-valley" galaxies is observed. While the actual nature of these sources is not definitely understood, the results of this work suggest that a significant fraction of faint radio sources might be composite, transition objects, thus a simple "AGN vs starforming" classification might not be appropriate to fully understand what faint radio populations really are.
\end{abstract}

Panoramic Radio Astronomy: Wide-field 1-2 GHz research on galaxy evolution - PRA2009

June 02 - 052009

Groningen, the Netherlands

\footnotetext{
${ }^{*}$ Speaker.

${ }^{\dagger}$ The National Radio Astronomy Observatory is a facility of the National Science Foundation operated under cooperative agreement by Associated Universities, Inc..
} 


\section{Introduction}

In recent years, many studies have agreed in assigning a relevant role to AGN feedback in shaping the evolution of galaxies, making the co-evolution of galaxies and AGNs a fundamental piece in the puzzle of the general evolution of galaxy populations. At the same time, deep radio surveys have been conducted in association with multiwavelength observations, allowing such (co)evolution of galaxies and massive black holes to be probed. These deep radio surveys, for the most part at $1.4 \mathrm{GHz}$, opened a window on the previously largely unexplored $\mu J y$ populations. However, unlike the Jy and mJy populations, which are dominated by radio loud AGNs hosted by quiescent galaxies, the $\mu J y$ population appears to be increasingly dominated by different kinds of sources, starforming galaxies and low-luminosity AGNs.

The individual contribution of AGNs and starforming galaxies to the whole $\mu J y$ population has proved difficult to determine accurately, and still is a matter of active investigation. Deep surveys coupling high quality radio imaging with a wide panchromatic coverage recently upgraded such investigation to a full multiwavelength approach (e.g. $[16,8,7,12])$. Together with the promise of the new upcoming facilities, this will certainly lead to a better understanding of the real nature of $\mu J y$ radio populations.

The AB magnitude system and WMAP cosmology $\left(\Omega_{M}=0.27, \Omega_{\Lambda}=0.73, \mathrm{H}_{0}=71 \mathrm{~km} \mathrm{~s}^{-1}\right.$ $\mathrm{Mpc}^{-1}$ ) are used in the following.

\section{A highly complete sample of distant faint radio sources}

In this work we exploit a $20 \mathrm{~cm}$ selected sample from a very deep $1.4 \mathrm{GHz}$ VLA image of the Deep Swire Field [11]. We take advantage of broad-band photometry available in up to 11 passbands from GALEX NUV to IRAC $4.5 \mu \mathrm{m}$, in order to estimate photometric redshifts and stellar population properties of the host galaxies [17]. In particular, the comparison of the observed spectral energy distributions (SEDs) with synthetic or semi-empirical templates allowed us to classify the radio sources as quiescent, intermediate, or starforming galaxies, from the point of view of the optical-NIR SED. As a reference, the restframe U-B color ranges for the three classes of quiescent, intermediate, and starforming objects are approximately 1.1-1.4, 0.9-1.1, 0.1-0.9, respectively, while the break strenghts at $4000 D_{n}(4000)$ are about 1.6-2.1, 1.5-1.7, 1-1.3.

We focus in the following on the sample at redshift $0.3<z<1.3$, which is estimated to be overall more than $90 \%$ complete in terms of counterparts and redshifts [17]. It is important to remind that, since the Deep Swire Field is imaged with a single VLA pointing, the rms varies as a function of distance from the image center and thus the whole radio sample is not a flux-limited sample. In the following we will restrict to flux-limited sub-samples when dealing with statistical properties of the radio sources.

\section{The nature of faint radio populations}

In this work we investigate the nature of faint radio sources from the point of view of their stellar populations. In fact, galaxies with a quiescent SED typical of a passively evolving stellar population are very likely to have most of their radio flux contributed by an AGN. On the other 
hand, galaxies with an SED showing ongoing star formation are likely to have at least some of their radio flux produced by such star formation activity. The comparison of the star formation rate as estimated by different indicators, such as the restframe UV and radio flux densities, yields further information on the actual contribution of star formation in powering the radio source. Nonetheless, it is important to remind that the optical-NIR SED of a galaxy may be affected by several factors (and most notably dust extinction) which may lead to a misclassification of the source in this scheme.

In figure 1 we show the radio luminosity as a function of redshift for all radio sources in the range $0.3<z<1.3$, divided by SED class. We remind the reader that due to the non-evolving nature of the templates used, figure 1 shows the stellar populations status (i.e. actively starforming, passively evolving, etc.) at the time of observations, without any evolutionary link between sameclass objects at different cosmic epochs.

As figure 1 shows, all the sub-classes of radio sources (but possibly the bluer starforming) span a wide range in the radio luminosities probed here. However, at all redshifts the highest radio luminosities in the probed range are typical of low-starforming systems (intermediate and

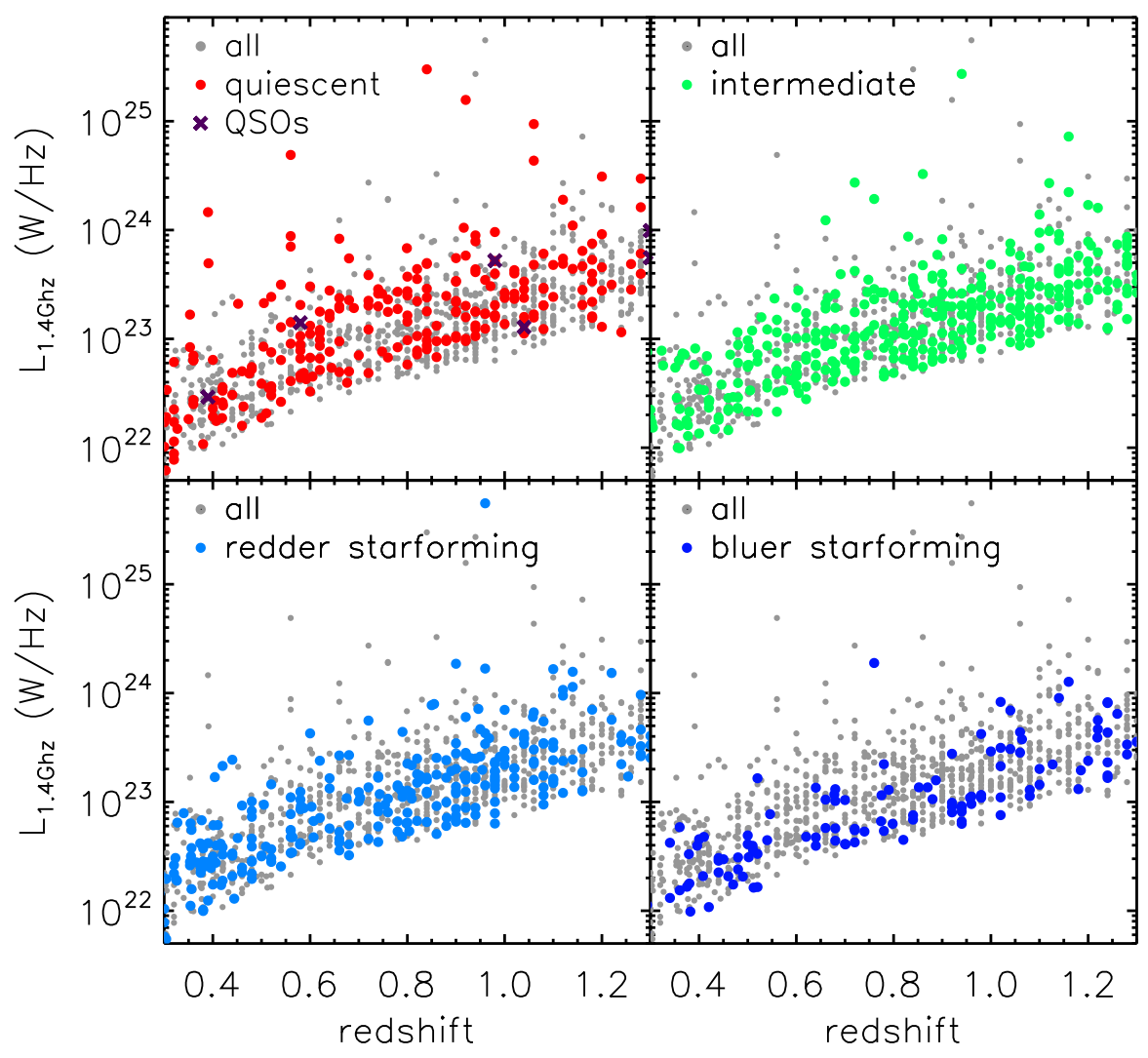

Figure 1: The 1.4GHz luminosity plotted against redshift, for objects with $0.3<z<1.3$. Grey points in all panels show all data points while colored symbols show objects whose photometry was best-fitted by SEDs of different types, as indicated in each panel. We note that in this figure the "starforming" class is further split in two sub-classes of starforming templates, "redder SF" and "bluer SF", with restframe U-B ranging in $0.7-0.9$ and $0.1-0.7$, and $D_{n}(4000)$ about $\approx 1.3,1-1.2$, respectively. 
quiescent galaxies), with starforming galaxies tending to avoid the highest radio luminosities, with just few exceptions. Typical radio luminosities of the bluer starforming are lower than the bulk of the redder starforming, and for the most part seem to lie just above the flux density limit of this survey.

Figure 1 also shows how a significant part of this radio galaxy sample is made of "intermediate" objects. This is more clear in figure 2 which shows the restframe U-B colors of our radio sample between redshift 0.3 and 1.3. The left-hand panel shows the color-magnitude diagram for the whole $0.3<z<1.3$ sample, with the points color-coded according to their SED class. The dotted line shows the separation between red sequence and blue cloud as defined for a optically selected sample [19]: of course galaxies classified as quiescent lie on the red sequence and starforming objects in the blue cloud. However, as shown in the flux-limited sub-samples in the right-hand panels, the peak of the restframe U-B distribution lies at the expected location of the division line between red and blue galaxies [19], in the so-called green valley. This is likely due to the depth of this survey which allows us to go beyond the AGN dominated population, but at the same time does not yet allow us to reach the still fainter radio luminosities more typical of the bulk of normal starforming systems.

We note that, as expected, the contribution of quiescent, intermediate and starforming systems changes with the radio flux limit. In the shallowest sub-sample considered $\left(S_{1.4 \mathrm{GHz}}>84 \mu \mathrm{Jy}\right)$, the fractions of galaxies classified as starforming, intermediate, and quiescent are roughly simi$\operatorname{lar}(33 \%, 36 \%, 30 \%$, respectively), but eventually they become $45 \%, 33 \%, 22 \%$ in the deepest sub-sample $\left(S_{1.4 \mathrm{GHz}}>16 \mu \mathrm{Jy}\right)$. This clearly suggests how the contributions of the actively starforming and quiescent galaxies change with the flux density limit of the sample, with quiescent AGN-dominated galaxies increasing their relevance in brighter samples and, viceversa, starforming galaxies becoming more important in fainter samples. We note that if instead of flux-limited
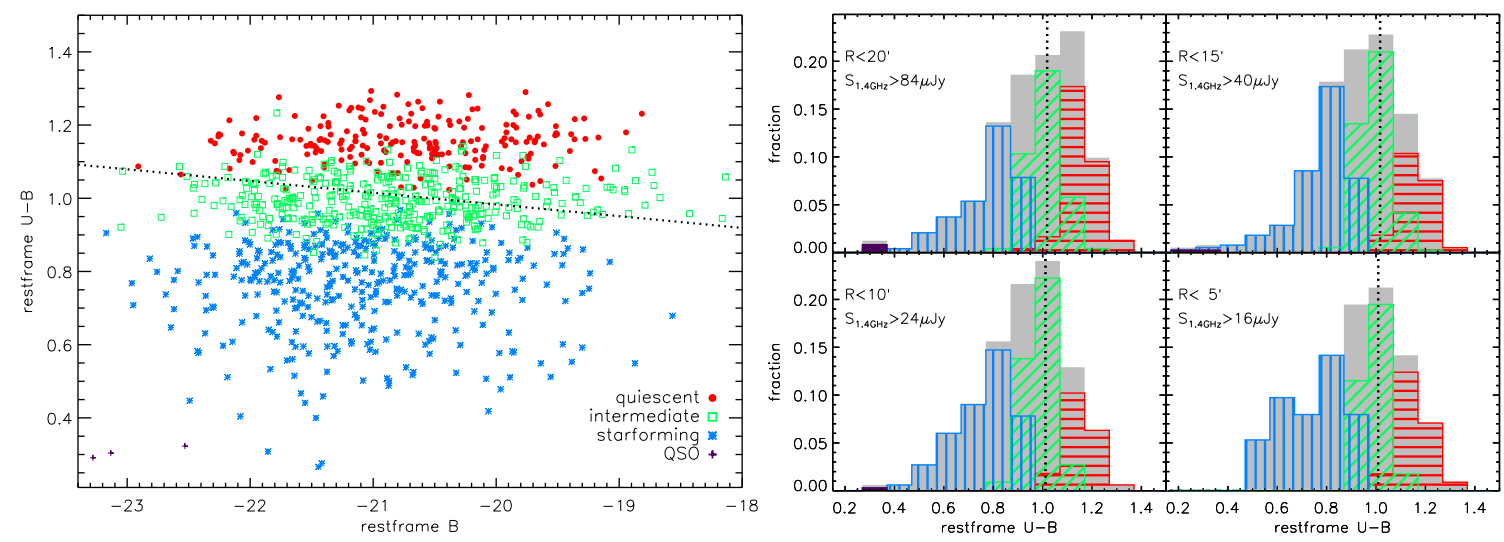

Figure 2: Left-hand panel: the restframe U-B vs B color-magnitude diagram for all sources with redshift $0.3 \leq z \leq 1.3$. Symbols are color coded according to best-fit SED type, as indicated. The dotted line shows the division between red sequence and blue cloud from DEEP2 data as in [19]. Right-hand panel: the distribution of restframe U-B color in four flux-limited sub-samples. The dotted lines mark the U-B color of the [19] division line at the median B magnitude of each plotted sub-sample. In each panel, the grayshaded histogram shows the distribution of the whole sub-sample while histograms for the different SED classes are color-coded as in the left-hand panel. 


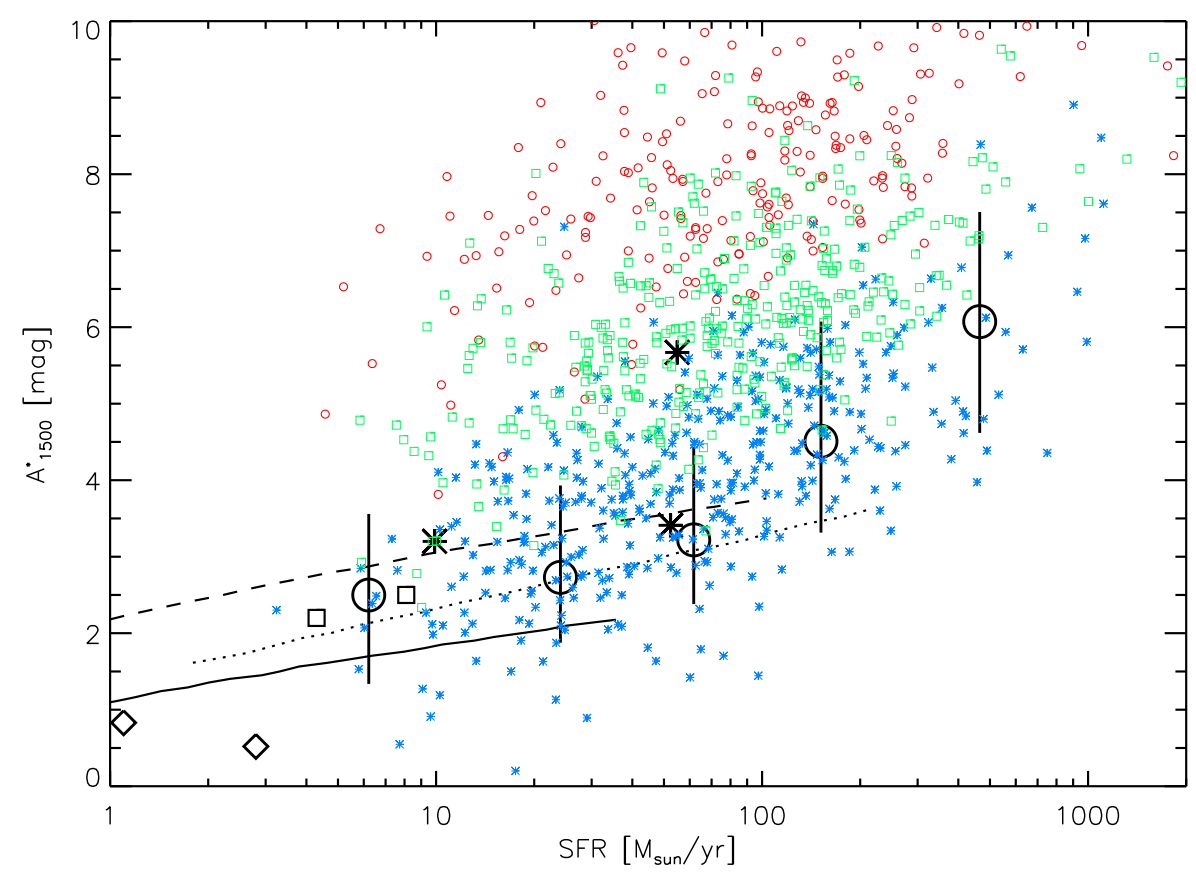

Figure 3: The "dust attenuation" $\mathrm{A}_{1500}^{*}=2.5 \mathrm{Log}\left(\mathrm{SFR}_{\text {radio }} / \mathrm{SFR}_{U V}\right)$ against "star formation rate" as determined from the $1.4 \mathrm{GHz}$ luminosity (see text for the actual meaning of the two quantities plotted). Small symbols color coded as in figure 2 show our $0.3<z<1.3$ radio sample, 34 objects (95\% of whom are red or green) with $\mathrm{A}_{1500}^{*}>10$ are not plotted. Large empty circles with errorbars show the [13] results (median and $16-84^{\text {th }}$ percentile range) for star forming BzK galaxies at $z \approx 2$. Empty squares, diamonds and stars show local galaxies from [2,3] (stars/diamonds symbols are for galaxies classified as dust-rich and dust poor, respectively). The solid, dotted and dashed lines show the dust attenuation as a function of SFR as derived from the local optical/UV-selected sample of [6], the intermediate redshift NIR/MIR-selected sample of [4] and the low-redshift radio-selected sample of [1], respectively.

samples we consider flux ranges this trend becomes, as expected, more evident: if considering just the faintest sources with flux density $16 \mu \mathrm{Jy}<S_{1.4 G H z}<24 \mu \mathrm{Jy}$ less than $10 \%$ are classified as quiescent while the fraction of actively starforming systems has increased to more than $50 \%$. The U-B color distribution of this faintest sample is different from that of the $S_{1.4 \mathrm{GHz}}>84 \mu \mathrm{Jy}$ flux-limited sample at a $>99.7 \%$ significance level. On the other hand, we also remind the reader of the different redshift distributions of populations of different flux density: less than $25 \%$ of the $16 \mu \mathrm{Jy}<S_{1.4 \mathrm{GHz}}<24 \mu \mathrm{Jy}$ sample is at $0.3<z<0.7$, compared to more than $40 \%$ of the $S_{1.4 \mathrm{GHz}}>84 \mu \mathrm{Jy}$ sample (see [17] for details).

As we mentioned above, the comparison of different star formation rate (SFR) indicators for the sources in our sample may help us disentangling AGN- from starformation-powered radio emission. We show one kind of such approach in figure 3, where we compare our classification of this radio sample with populations of starforming galaxies with regard to their radio/UV flux densities. Figure 3 shows for our sample the SFR as determined from the $1.4 \mathrm{GHz}$ luminosity against the dust attenuation estimated as $\mathrm{A}_{1500}^{*}=2.5 \mathrm{Log}\left(\mathrm{SFR}_{\text {radio }} / \mathrm{SFR}_{U V}\right)$. We note that the meaning of both quantities is not well defined for the whole sample. In fact, only for galaxies whose radio emission is due to star formation the quantity plotted as "SFR" actually represents the SFR, and 
$\mathrm{A}_{1500}^{*}$ indeed is the dust attenuation. Instead, galaxies with AGN contribution are expected to have their $1.4 \mathrm{GHz}$ luminosity at least partially contaminated by the AGN, and thus both SFR and $\mathrm{A}_{1500}^{*}$ lose their meaning for these objects. Keeping this in mind, this plot allows a simple comparison to be made between our sample and populations of star forming galaxies, at different redshifts and selected with different criteria (UV, optical, IR, radio). As the figure shows, the range of attenuations derived for our starforming objects is in very good agreement with other studies of star forming galaxies $[1,2,3,4,6,13]$. On the other hand, while part of the objects classified as intermediate would also overlap with these samples, it is clear how the $A_{1500}^{*}$ for the intermediate and, even more significantly, for the quiescent sources, generally lies above the expectations, either suggesting that these populations exhibit unusually high extinctions as compared to their SFRs, or supporting the idea that a significant fraction of their radio luminosity is contributed by AGN, thus making the SFR and $\mathrm{A}_{1500}^{*}$ estimates meaningless. Even though part of the intermediate sources might still be very dusty starforming objects, it is useful to remind here that a significant occurrence of AGN hosts among the green valley population, as well as their nature of composite (starforming+AGN), transition systems, has been noted in several previous studies (e.g., [5,9,10,14,15,18]). Folding in the available (and upcoming) X-ray and IR information will allow us to better understand the actual nature of these sources.

\section{References}

[1] Afonso, J., Hopkins, A., Mobasher, B., \& Almeida, C. 2003, ApJ, 597, 269

[2] Calzetti, D. 2001, PASP, 113, 1449

[3] Calzetti, D., Armus, L., Bohlin, R. C., et al. 2000, ApJ, 533, 682

[4] Choi, P. I., Yan, L., Im, M., et al. 2006, ApJ, 637, 227

[5] Choi, Y.-Y., Woo, J.-H., \& Park, C. 2009, ApJ, 699, 1679

[6] Hopkins, A. M., Connolly, A. J., Haarsma, D. B., \& Cram, L. E. 2001, AJ, 122, 288

[7] Huynh, M. T., Jackson, C. A., Norris, R. P., \& Fernandez-Soto, A. 2008, AJ, 135, 2470

[8] Mainieri, V., Kellermann, K. I., Fomalont, E. B., et al. 2008, ApJS, 179, 95

[9] Martin, D. C., Wyder, T. K., Schiminovich, D., et al. 2007, ApJS, 173, 342

[10] Nandra, K., Georgakakis, A., Willmer, C. N. A., et al. 2007, ApJL, 660, L11

[11] Owen, F. N. \& Morrison, G. E. 2008, AJ, 136, 1889

[12] Padovani, P., Mainieri, V., Tozzi, P., et al. 2009, ApJ, 694, 235

[13] Pannella, M., Carilli, C. L., Daddi, E., et al. 2009, ApJL, 698, L116

[14] Salim, S., Rich, R. M., Charlot, S., et al. 2007, ApJS, 173, 267

[15] Schawinski, K., Thomas, D., Sarzi, M., et al. 2007, MNRAS, 382, 1415

[16] Smolčić, V., Schinnerer, E., Scodeggio, M., et al. 2008, ApJS, 177, 14

[17] Strazzullo, V., Pannella, M., Owen, F., et al. 2009, ApJ submitted

[18] Wild, V., Kauffmann, G., Heckman, T., et al. 2007, MNRAS, 381, 543

[19] Willmer, C. N. A., Faber, S. M., Koo, D. C., et al. 2006, ApJ, 647, 853 